\title{
EDUCAÇÃO VIRTUAL COMO AGENTE TRANSFORMADOR DOS PROCESSOS DE APRENDIZAGEM
}

\section{EDUCACIÓN VIRTUAL COMO AGENTE TRANSFORMADOR DE LOS PROCESOS DE APRENDIZAJE}

\author{
VIRTUAL EDUCATION AS A TRANSFORMING AGENT OF THE LEARNING \\ PROCESSES
}

\author{
Katihuska MOTA ${ }^{1}$ \\ Carlos $\mathrm{CONCHA}^{2}$ \\ Natalie MUÑOZ ${ }^{3}$
}

RESUMEN: En el presente artículo de tiene como objetivo analizar la educación virtual como agente transformados de los procesos de aprendizaje a través de una investigación documental y descriptiva para la cual se toman como referencia las estadísticas que proporciona el ministerio de educación de Chile, Perú y Colombia en relación al uso de las tecnologías de información y comunicación (TIC's) en la educación bien sea como apoyo académico en sus clases presenciales o como herramienta pedagógica de clases virtuales. Luego del desarrollo de la misma se tuvo como resultado con el paso de los años la implementación de la educación virtual se hace más común, aportando alternativas de enseñanza y aprendizaje eficaces que colaboran en las instituciones educativas a la formación de individuos competentes mediante el aprendizaje significativo.

PALABRAS CLAVE: Educación. Aprendizaje. Trasformación. Tecnología. Educación virtual.

RESUMO: Este artigo tem como objetivo analisar a educação virtual como agente transformadora dos processos de aprendizagem por meio da pesquisa documental $e$ descritiva, para a qual as estatísticas fornecidas pelo Ministério da Educação do Chile, Peru e Colômbia em relação ao uso das tecnologias de informação e comunicação (TICs) na educação, seja como suporte acadêmico em suas aulas presenciais, seja como ferramenta pedagógica para aulas virtuais. Após seu desenvolvimento, a implantação da educação virtual tornou-se mais comum com o passar dos anos, proporcionando alternativas eficazes de ensino e aprendizagem que colaboram nas instituições de ensino para formar indivíduos competentes por meio de aprendizagens significativas.

\footnotetext{
${ }^{1}$ Universidad Miguel de Cervantes (UMC), Chile. Doutora em Ciências da Educação, Mestre em Gestão de Empresas linha Operações, Engenheiro de Petróleo. ORCID: http://orcid.org/0000-0002-4108-957X. E-mail: motakt@gmail.com

${ }^{2}$ Universidad Miguel de Cervantes (UMC), Chile. Mestre em Educação. Engenheiro em Administração de Empresas linha Finanças. ORCID: https://orcid.org/0000-0002-5155-9694. E-mail: concharojascarlos@gmail.com

${ }^{3}$ Universidad Miguel de Cervantes (UMC), Chile. Doutorando em educação, Mestre em Educação, Professora de Ciências menção Biologia, Licenciada em Educação, Acadêmica, Direção de Pós-graduação e Pesquisa. ORCID: http://orcid.org/0000-0002-5430-1517.E-mail: natalie.munoz@profe.umcervantes.cl
}

RPGE- Revista on line de Política e Gestão Educacional, Araraquara, v. 24, n. 3, p. 1216-1225, set./dez. 2020. e-ISSN:1519-9029. 
PALAVRAS-CHAVE: Educação. Aprendizagem. Transformação. Tecnologia. Educação virtual.

ABSTRACT: In this article, the aim is to analyze virtual education as a transformed agent of learning processes through documentary and descriptive research, for which the statistics provided by the Ministry of Education of Chile, Peru and Colombia in relation to the use of information and communication technologies (ICTs) in education either as academic support in their face-to-face classes or as a pedagogical tool for virtual classes. After its development, the implementation of virtual education became more common over the years, providing effective teaching and learning alternatives that collaborate in educational institutions to train competent individuals through meaningful learning.

KEYWORDS: Education. Learning. Transformation. Technology. Virtual education.

\section{Introdução}

O ensino on-line é um elemento que permite desenvolver o processo de ensino e aprendizagem através da implementação das tecnologias da informação e comunicação (TIC's), assim, se pode realizar o trabalho educacional a partir de qualquer lugar sem necessidade de se encontrar de forma presencial com o professor ou docente. Esta permite adquirir habilidades e conhecimentos a ritmo próprio e não de forma coletiva, em um tempo próprio pré-determinado pelo estudante e não limitado a um horário, esta metodologia faz com que o estudante se torne, na maioria dos casos, um autodidata.

Chamamos aqui de educação virtual um sistema aberto que é guiado pelo usuário, neste se promove o intercâmbio de ideias e conhecimento através de diferentes espaços com o guia e apoio de um facilitador que será o professor que dirige a atividade educacional. Por ser um sistema de ensino não presencial compreende diversas atividades e tarefas que não se visualizam nas aulas tradicionais, tal é o caso do papel ativo do estudante para a construção de significados e a resolução de problemas em casos reais, construção de mapas mentais e organizadores gráficos e o intercâmbio de ideias para compreender os objetivos das disciplinas.

Nos processos de aprendizagem, a interação social é muito importante devido a que a maioria do que apreendemos provém do contato com outras pessoas, por tanto, na educação virtual é fundamental o uso de chat, vídeo chamadas, videoconferências, fóruns como ferramentas de comunicação síncrona e o e-mail, plataformas digitais e mensagens como ferramentas de comunicação assíncrona. A base neste processo de aprendizagem é o trabalho 
colaborativo grupal, onde se dá o intercâmbio de experiências para a resolução de problemas (HERNÁNDEZ, 2017).

No entanto, algumas pessoas pensam que a interação social através das plataformas virtuais não é suficiente no processo de aprendizagem, cabe destacar que existem muitas ferramentas úteis como os simuladores que tributam a melhorar a aprendizagem e permite que este seja significativo, de modo que as plataformas de aprendizagem permitem a incorporação de vídeos explicativos onde se podem realizar experimentos simples e reaplicáveis em qualquer contexto como ferramenta de aprendizagem eficaz.

Portanto, o uso do ensino on-line vem a ser um agente transformador das aprendizagens que cada dia se integra da melhor forma às atividades cotidianas nas escolas. Parra (2012) assegura que a tecnologia teve uma grande influência a nível educacional sendo uma ferramenta útil nos processos de aprendizagem e tem se transformado em parte da "cotidianidade escolar". Diante do exposto, se pode dizer que a educação virtual permite que se deem processos de aprendizagem significativa através da implementação da tecnologia como instrumento educacional que permite melhorar a qualidade do estudante.

De acordo com o exposto por Granados (2015), os recursos tecnológicos tem se transformado em recursos educacionais para facilitar a aprendizagem, rompendo os meios tradicionais de aprendizagem e proporcionando abordagens, assumindo novos desafios no trabalho de ensino e, portanto, no processo de aprendizagem. É desse modo que o ensino online permite a geração de competências que são requeridas pela sociedade e depende da habilidade do docente criar o ambiente ideal para a aprendizagem significativa.

A educação virtual vem a ser um agente transformador dos processos de aprendizagem à medida que rompe com as pautas da educação tradicional e incorpora não apenas o trabalho colaborativo, mas também aplica as TIC's como uma ferramenta que, além de transmitir informação permite aplica-la em diferentes contextos e modelar a diversidade de dados para a verificação de estratégias na sistematização dos processos, com o qual se desenvolvem competências cognitivas onde o estudante é um agente educacional com critério e pensamento próprio.

A empresa Intel Educación (2018) apresentou um guia útil no qual Robert Gravina, chefe de informática e tecnologia, expõe que as tecnologias móveis permitem melhorar os resultados acadêmicos através de:

Soluções de produtividade que dão aos professores mais tempo para trabalhar com os alunos e permitem que as escolas tenham um desempenho mais eficaz. Portais seguros que estendem o dia escolar, melhoram a 
comunicação entre escola e casa e dão aos pais a oportunidade de se tornarem parceiros ativos. Tecnologias colaborativas que ajudam os professores a reduzir o isolamento e expandir seus conhecimentos, participando de comunidades de desenvolvimento profissional (INTEL EDUCACIÓN, 2018, p. 72). ${ }^{4}$

Diante do exposto, pode-se dizer que os recursos digitais, não apenas móveis senão a nível geral permitem melhorar a didática das aulas incorporando vídeos, imagens e um alto nível de interatividade que permitem a melhor compreensão dos conteúdos das disciplinas e que se transformam em potentes fontes de busca de informação complementando os processos investigativos de docentes e estudantes de todos os níveis educacionais.

A educação virtual proporciona uma abordagem real e importante para o desenvolvimento das habilidades, capacidades e destrezas de docentes e estudantes para que o processo de ensino seja eficaz. O mais importante é que se possa fazer uso formativo dos meios que gerem experiências significativas de aquisição de conhecimentos.

García e Muñoz (2013), citam a Unesco, indicando que esta estabelece várias diretrizes que permitem potencializar a aprendizagem através do uso da tecnologia e inclusive proporcionam capacitação aos docentes para criar conteúdos e blogs, além de ampliar as opções de conectividade através da elaboração de estratégias que promovem o uso seguro e responsável das ferramentas tecnológicas.

Neste contexto, surgem algumas perguntas: a educação virtual é realmente um fator dos processos de aprendizagem? A educação virtual poderia tirar o lugar das aulas presenciais? Para responder a estes questionamentos se tem a opinião de Granados (2015), quem indica que as aulas que se desenvolvem em sala de aula, ou seja, de forma presencial devem gerar espaços que se completem com a tecnologia, portanto, tanto os docentes como os estudantes devem ir com de mãos dadas com o crescimento tecnológico e atualizar seus conhecimentos com relação às TIC's para adquirir habilidades que lhes permita o crescimento pessoal, educacional, laboral, e, em linhas gerais, lhe permite melhorar a interação social.

A educação virtual permite a geração de diferentes cenários que permitem tanto a docentes, estudantes como as instituições educacionais em geral o aproveitamento das oportunidades e a geração de espaços que permitam melhorar o processo de ensino e aprendizagem em contextos sociais e de trabalho colaborativo que se adaptam à sociedade

\footnotetext{
${ }^{4}$ Soluciones de productividad que brindan a los maestros más tiempo para trabajar con los estudiantes y permiten a las escuelas desempeñarse con mayor eficacia. Portales seguros que amplíen la jornada escolar, mejoren la comunicación entre la escuela y el hogar y de oportunidad a los padres de familia a convertirse en colaboradores activos Tecnologías de colaboración que ayuden a los maestros a reducir el aislamiento y ampliar sus conocimientos tomando parte en comunidades de preparación profesional (INTEL EDUCACIÓN, 2018, p. 72).
} 
atual. Para autores como Díaz-Barriga (2013) e Tapia e León (2013), há uma série de diretrizes que apoiam a inclusão da tecnologia na educação, a primeira das quais é o fácil acesso à informação, maior abrangência e melhor comunicação e, finalmente, a geração de habilidades que permitem aos estudantes e professores se adaptarem aos desafios impostos pela globalização.

É desse modo que a educação virtual pode ser definida como o processo educacional (que envolve tanto o ensino como a aprendizagem) que se dá mediante o uso da tecnologia e que em ocasiões, depende do alcance que possui, deixando de lado as aulas presenciais. Esta educação virtual pode se dar de duas formas: sem a presença de um docente, através de uma plataforma de respostas padronizadas ou com a orientação de um docente com o qual os estudantes podem participar de forma contínua em diferentes seções.

A nível de gestão educacional se visualiza a educação virtual como um processo que se realiza mediante a combinação de uma série de tarefas sistemáticas que se encontram distribuídas em 4 áreas:

- Organizacional, corresponde com o seguimento e controle dos processos de formação tanto de docentes (para sua formação em gestão de recursos) como os estudantes (para a correta utilização dos recursos didáticos e de avaliação), ou seja, tem a ver com a prática institucional.

- Tecnológica, é a ferramenta través da qual se dão os processos de comunicação, docente-estudante e estudante-docente, além disso, possui diversidade de instrumentos que servem de apoio para o desenvolvimento das aulas.

- Educacional, é a área que permite a criação dos planos de estudo, objetivos das disciplinas, distribuição do conteúdo, entre outros elementos que colaborem para que o processo de ensino e aprendizagem se dê de forma satisfatória.

- Impacto social, é o que compreende os valores e opiniões da práxis educacional na educação virtual.

Para Granados (2015), a educação virtual contribui para a transformação do processo educacional porque envolve um processo educacional amplo e de longo alcance que deve ser controlado através de diferentes sistemas que respondem por sua contribuição à prática educacional, é um campo de emprego de agentes inovadores para a construção do conhecimento, proporcionando autenticidade ao aprendizado.

Como a pesquisa é documental, uma série de estatísticas é utilizada que permitem uma abordagem na análise descritiva. Neste sentido, para investigar este tema, foram selecionadas estatísticas do Ministério da Educação de diferentes países da América Latina (neste caso, 
Chile, Peru e Colômbia foram selecionados como referências, dado que as informações estão disponíveis nestes países), o que permitirá conhecer o progresso do processo de mudança do ensino presencial para o virtual.

A comparação dos dados que serão tomando as porcentagem de uso de cada uma das modalidades de educação em diferentes países para posteriormente realizar uma análise com as médias de cada uma das porcentagens obtidas, isso com o fim de conhecer a frequência de uso da educação virtual e a vinculação que experimentou nos últimos 5 anos.

Inicialmente, apresentamos abaixo: Tabela 1, Tabela 2, Tabela 3, onde se evidenciam os valores percentuais com relação à modalidade da educação no Chile, Peru e Colômbia respectivamente.

Tabela 1 - Estatísticas sobre o Progresso da Educação Virtual Chile.

A partir de 2015-2019

\begin{tabular}{|c|c|c|}
\hline Ano & $\begin{array}{c}\text { Estatística Educação } \\
\text { Presencial }\end{array}$ & Estatística Educação Virtual \\
\hline 2015 & $83,2 \%$ & $16,8 \%$ \\
\hline 2016 & $78,5 \%$ & $21,5 \%$ \\
\hline 2017 & $69,3 \%$ & $30,7 \%$ \\
\hline 2018 & $60,1 \%$ & $39,9 \%$ \\
\hline 2019 & $55,6 \%$ & $44,4 \%$ \\
\hline Média & $\mathbf{6 9 , 3} \%$ & $\mathbf{3 0 , 7} \%$ \\
\hline
\end{tabular}

Fonte: Elaboração própria tomando por base os dados do Ministério da Educação Chile.

Ao observar a tabela 1 se tem que, com o passar dos anos, se apresenta um aumento entre $8 \%$ e $10 \%$ no uso da educação virtual, o qual entrega uma média de $30,7 \%$ para os 5 anos, resultado significativo considerando que os valores aumentam com o passar dos anos, significando que a virtualidade e o uso da tecnologia na educação se encontra apresentando grandes contribuições para a educação atual.

Tabela 2 - Estatísticas de Avanços da Educação virtual Peru.

Desde 2015-2019

\begin{tabular}{|c|c|c|}
\hline Ano & Estatística Educação Presencial & $\begin{array}{c}\text { Estatística } \\
\text { Educação Virtual }\end{array}$ \\
\hline 2015 & $91,2 \%$ & $8,8 \%$ \\
\hline 2016 & $85,3 \%$ & $14,7 \%$ \\
\hline 2017 & $80,1 \%$ & $19,9 \%$ \\
\hline 2018 & $75,8 \%$ & $24,2 \%$ \\
\hline 2019 & $70,1 \%$ & $29,9 \%$ \\
\hline Promedio & $\mathbf{8 0 , 5} \%$ & $\mathbf{1 9 , 5} \%$ \\
\hline
\end{tabular}

Fonte: Elaboração própria tomando como base os dados do Ministério da Educação Peru. 
Ao visualizar a tabela 2, se evidenciava que Peru apresenta aumentos que oscilam entre $4 \%$ e $6 \%$, cifra significativamente menor com respeito à apresentada pelo Chile, mas que do mesmo modo leva a um aumento do uso da educação virtual no Peru, o qual é vantajoso para o país, apresentando elementos de aprendizagens de longo alcance e que não estão essencialmente baseados na presencialidade educacional.

Tabela 3- Estatística de Avanços da Educação virtual Colômbia. Desde 2015-2019.

\begin{tabular}{|c|c|c|}
\hline Ano & $\begin{array}{c}\text { Estatística Educação } \\
\text { Presencial }\end{array}$ & $\begin{array}{c}\text { Estatística } \\
\text { Educação Virtual }\end{array}$ \\
\hline 2015 & $80,8 \%$ & $19,2 \%$ \\
\hline 2016 & $71,5 \%$ & $28,5 \%$ \\
\hline 2017 & $70,3 \%$ & $29,7 \%$ \\
\hline 2018 & $61,1 \%$ & $38,9 \%$ \\
\hline 2019 & $52,3 \%$ & $47,7 \%$ \\
\hline Promedio & $\mathbf{6 7 , 2} \%$ & $\mathbf{3 2 , 8} \%$ \\
\hline
\end{tabular}

Fonte: Elaboração própria tomando como base os dados do Ministério de Educação Colômbia.

$\mathrm{Na}$ tabela 3, se apresentam as porcentagens de avanço da educação virtual para Colômbia, cifras que claramente vão aumentando com o passar dos anos com um aumento de 9\% em quase todos os anos, a exceção do ano 2016 e 2017, cujo aumento foi muito pouco, evidenciando que por problemas de reformas educacionais se detiveram os avanços em quanto à educação virtual e posteriormente contínuo ganhando terreno esta última com respeito à educação presencial.

É importante destacar que, nos países apresentados se observa um aumento impressionante na implementação da educação virtual, para confirmar estes valores se faz uma análise geral das médias anteriores e se tem os resultados apresentados no gráfico 1 que se mostra a seguir. No qual se comparam as médias dos países tomados como referência no período 2015-2019 para uma análise mais profunda quanto à transcendência da educação virtual sobre a educação presencial. 
Figura 1 - Gráfico da Comparação de Avanços da Educação virtual entre Chile, Peru e Colômbia para os anos 2015-2019.

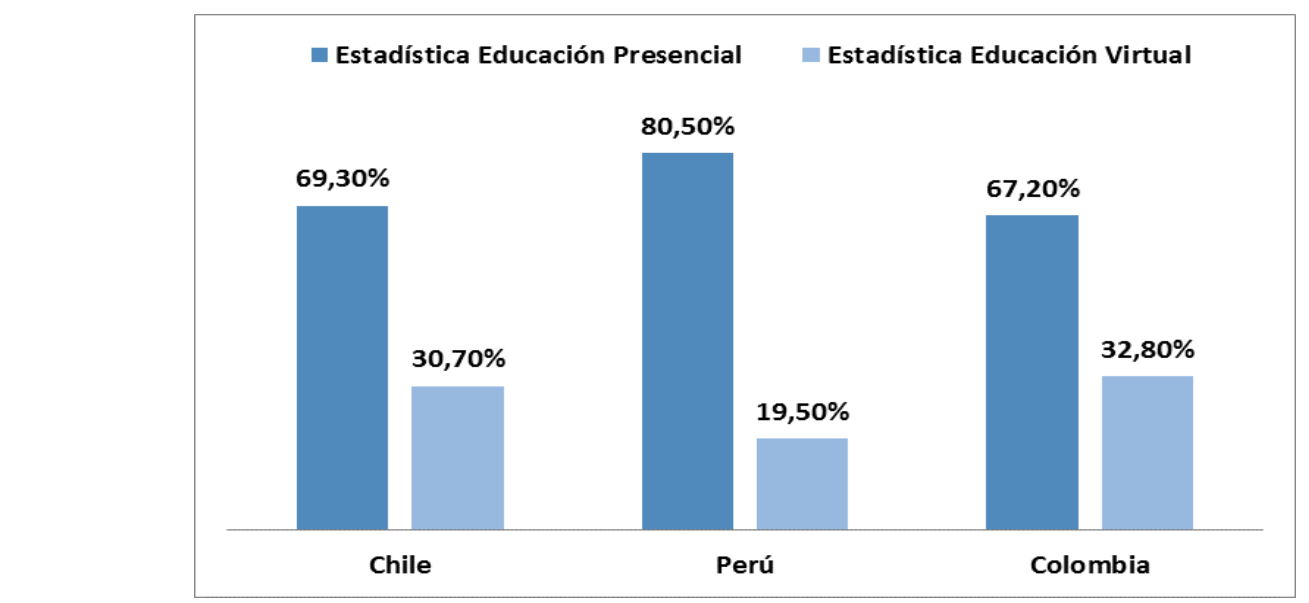

Fonte: Elaboração própria

Na figura 1, se observa que o Peru é o país que mais utiliza a educação presencial, diferentemente do Chile e da Colômbia que experimentaram um crescimento no uso da educação virtual em seus processos de aprendizagem. Fazendo um prognóstico dos resultados obtidos se pode dizer que nos próximos 5 anos a educação virtual terá uma participação significativa nos processos de aprendizagem com um posicionamento igual ou maior na educação presencial, isso motivado com as vantagens que se observou e os avanços que permitiram obter não só a nível de comunicação, mas também a nível de aquisição de conhecimentos.

\section{Considerações finais}

Uma vez desenvolvido o artigo, é necessário que a educação virtual forneça elementos positivos no processo de ensino e aprendizagem. Ela também aumenta o interesse na aquisição de conhecimento, uma vez que utiliza vídeos e imagens que contribuem para o processo de aprendizagem significativa, além de proporcionar novas experiências de aprendizagem através do reforço do conhecimento, colocando problemas e entregando possíveis soluções.

Rojas (2013), fornece uma análise em relação à implementação da tecnologia para o aprendizado autônomo e destaca que a tecnologia muda a educação tradicional unidirecional para um sistema bidirecional onde todos aprendem através do diálogo interativo, além disso, indica que com o uso da tecnologia de ensino segue mantendo a segunda ordem de importância e o que é realmente protagonista é a aprendizagem e, além disso, estabelece que 
alguns países, a pesar dos avanços tecnológicos, resistem à implementação das TIC's nos processos educativos.

Após apresentar os casos de três países latino-americanos, é possível visualizar que o mundo está avançando a passos largos e se consolidando em uma educação virtual como protagonista dentro da gestão educacional, contribuindo com elementos significativos para os processos de ensino e transformando a presença da educação em ambientes digitais, que são implementados não apenas como instrumentos de apoio, mas também como base para o ensino de uma diversidade de disciplinas nos níveis secundário, técnico e universitário.

A incorporação da tecnologia no processo de aprendizagem promove ações para processos de treinamento e pesquisa e contribui para a socialização e compreensão do conteúdo em um grande número de disciplinas na prática educacional. Portanto, a educação virtual foi inserida no contexto educacional de forma permanente e é implementada para a geração de estratégias que fortalecem a socialização da informação nas disciplinas e também permite vincular esta informação com o contexto social no qual estudantes e professores estão imersos.

Finalmente, pode-se dizer que a educação virtual é um agente transformador dos processos de aprendizagem porque tem participação ativa dentro do processo de treinamento, permite uma aprendizagem autônoma e responde às exigências da realidade educacional na qual os indivíduos buscam capacitar sua aprendizagem em seu próprio tempo e espaço, o que lhes permite ter autocontrole e auto direcionamento, sendo estudantes independentes em sua aprendizagem e interdependentes, compartilhando conhecimento na aprendizagem colaborativa.

Assim, a educação que ocorre em ambientes virtuais ou utiliza ambientes virtuais como suporte na formação dos estudantes permite a geração de conhecimento através de uma aprendizagem significativa e com uma estratégia de trabalho colaborativo em que professores, estudantes e tecnologia se tornam uma tríade que privilegia o sistema educacional. Estes elementos permitirão que o modelo de formação pedagógica seja baseado no construtivismo, ou seja, é um modelo onde os indivíduos são capazes de gerar seu próprio senso de conhecimento.

\section{REFERÊNCIAS}

DÍAZ-BARRIGA, F. TIC en el trabajo del aula. Impacto en la planeación didáctica. Revista Iberoamericana de Educación Superior, v. 4, n. 10, p. 3-21. 
GARCÍA, A; MUÑOZ, R. Recursos digitales para la mejora de la enseñanza y el aprendizaje. Universidad de Salamanca, 2013. 58 p.

GRANADOS, A. Las TIC en la enseñanza de los métodos numéricos. Sophia Educación, v. 11, n. 2, p. 143-154, 2015.

HERNÁNDEZ, R. M. Impacto de las TIC en la educación: Retos y Perspectivas. Propósitos y Representaciones, v. 5, n. 1, p. 325-347, 2017.

INTEL EDUCACIÓN. Cómo trasformar la educación para la nueva generación. Guía práctica de la enseñanza-aprendizaje con tecnología. Chile, 2018

MICHELI J Y ARMENDÁRIZ, S. Estructuras de Educación Virtual en la Organización Universitaria. Formación Universitaria, México, v. 4, n. 6, p. 35-48, 2011.

PARRA, C. TIC, conocimiento, educación y competencias tecnológicas en la formación de maestros. Revista Nómadas, Bogotá (Colombia), n. 36, p. 145-159, 2012.

ROJAS, J. Educación virtual: del discurso teórico a las prácticas pedagógicas en la educación superior colombiana. 2013. Tesis (Doctoral) - Universidad Nacional de Educación a Distancia, Madrid, 2013.

TAPIA, E.; LÉON, J. Educación con TIC para la sociedad del conocimiento. Revista Digital Universitaria, México v. 14, n. 2, 2013.

UNESCO. Directrices para las políticas de aprendizaje móvil. Organización de las Naciones Unidas para la Educación, la Ciencia y la Cultura. París, Francia, 2013.

\section{Como referenciar este artigo}

MOTA, K.; CONCHA, C.; MUÑOZ, N. Educação virtual como agente transformador dos processos de aprendizagem. Revista on line de Política e Gestão Educacional, Araraquara, v. $24, \quad$ n. 3 , p. 1216-1225, set./dez. 2020. e-ISSN:1519-9029. DOI: https://doi.org/10.22633/rpge.v24i3.14358

Submetido em: 20/06/2020

Revisões reqeuridas: 18/07/2020

Aprovado em: 30/08/2020

Publicado em: 01/09/2020 